\begin{tabular}{|c|c|c|}
\hline 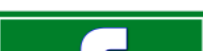 & Int.J.Curr.Microbiol.App.Sci (2021) 10(08): 620-628 & \\
\hline & $\begin{array}{l}\text { International Journal of Current Microbiology and Applied Sciences } \\
\text { ISSN: 2319-7706 Volume } \mathbf{1 0} \text { Number } 08 \mathbf{( 2 0 2 1 )} \\
\text { Journal homepage: } \underline{\text { http://www.ijcmas.com }}\end{array}$ & 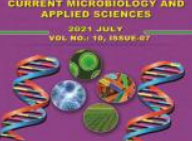 \\
\hline $\begin{array}{l}\text { EXCELLENT } \\
\text { PUBLISHERS }\end{array}$ & & \\
\hline
\end{tabular}

Review Article

https://doi.org/10.20546/ijcmas.2021.1008.072

\title{
Pernicious Combination of Covid-19 and Mucormycosis
}

\author{
Vijayalakshmi Nayak $^{1}$ and R. Shyamala ${ }^{2 *}$ \\ ${ }^{1}$ Department of Dermatology, ${ }^{2}$ Department of Microbiology, AIIMS Bibinagar, \\ Hyderabad, Telangana, India \\ *Corresponding author
}

A B S T R A C T

\section{Keywords}

Aspergillosis, acute respiratory distress syndrome, pneumonia

Article Info

Accepted:

25 July 2021

Available Online:

10 August 2021
During the pandemic, the combination of covid 19 with systemic steroids has caused a dramatic increase in the Mucormycosis cases in India. Our narrative review aims to understand the possible pathomechanisms involved in this exponential increase of Mucormycosis cases. The pathogen's virulence depends on the organism's ability to survive in the host by evading the immune system and causing harm to the host cells. Factors involved in the pathogenesis of COVID 19 which might be interested in the pathomechanisms of Mucormycosis are (1) increased expression of GRP 78 receptors (2) alteration of iron metabolism - the role of ferritin and iron (3) hepcidin mimicking activity (4) endothelities (5) damage to beta islet cells of pancreas (6) use of corticosteroid. Heath care-related or nosocomial infection though rare, can not be neglected. Mucormycosis developed in patients with both active SAR-CoV infection and patients who had recovered from COVID19 illness. Mucormycosis involving the nose and sinuses was the most common type. Early diagnosis and treatment of Mucormycosis are essential, as it improves the outcome and decreases the need for surgical intervention hence disfigurement. The combination of COVID 19 infection, steroid use, diabetes, and hospitalisation has caused a surge in Mucormycosis cases in COVID 19 infected patients. Further understanding of the pathogenesis helps in taking measures in the prevention and early management of the infection.

\section{Introduction}

Mucormycosis is a fatal angioinvasive fungal infection caused by Mucorales, which is having a very high mortality rate. Mucormycosis was first described in 1876 in a patient who died of cancer in Germany. The Past two decades saw an increase in the number of cases, more so in India and China
$(1,2)$. In India, it is estimated that the prevalence of Mucormycosis was 80 times more compared to the developed countries. (3) This increase in the prevalence can be partly attributed to the high prevalence of the diabetic population in these countries.(4)It is estimated that the prevalence of diabetes in India could rise to 101.2 million by 2030 . Since the pandemic, the combination of covid 
19 with systemic steroids has caused a dramatic increase in the Mucormycosis cases in India besides aspergillosis and candidiasis. Our narrative review aims to the understand the possible pathomechanisms involved in this exponential increase of Mucormycosis cases.

COVID-19 pandemic is caused by severe acute respiratory syndrome virus (SARS-CoV2 ). It is characterized by acute respiratory distress syndrome, pneumonia, and eventually multi-organ failure. About 180 million cases and 3.8 million deaths have been reported to the present day. Several treatment options are being evaluated, among which systemic steroids have been shown to improve the survival of the patients. Overzealous and unchecked systemic steroid use in COVID 19 patients is a crucial predisposing factor Mucormycosis development. COVID-19 infection induces damage of the alveolar epithelium and endothelialitis, hemoglobinopathy, cell iron overload, hypoxia, dysregulated immune system.(5-7). In the lung and immune dysfunction setting induced by the covid, widespread use of systemic corticosteroid, interleukin antagonists like tocilizumab and other comorbidities lead to the dramatic increase in the cases of Mucormycosis.

Social media and the press have speculated and emphasized the various other risk factors like steaming, long-term use of antibiotics, industrial contamination of oxygen cylinders, contaminated water used in the humidifier as a cause of the rise in the Mucormycosis cases. But there is no concrete evidence to support the speculations (8).

Mucorales areubiquitous organisms, and they are present in the beds, floors, side tables of hospitals. Regular decontamination of the surface and frequent hand washing should be emphasized to decrease the risk of nosocomial infection. Oxygen humidifier generates aerosols that can reach deep into the lung, due to which there is a risk of transmission of potential pathogens.(9) Reusable oxygen humidifiers get easily contaminated and should be replaced with a disposable humidifier to avoid nosocomial infection.

Antimicrobials have been used in the treatment of COVID 19. In a review, it was found that $72 \%$ of the patients were prescribed broad spectrum antibiotics. (10) Presently, there is no evidence to show whether prolonged antibiotics are a risk for Mucormycosis development.

\section{Causative agents}

The order Mucorales consist of 261 species, among which 38 cause human infection (11). Mucorales are typically thin-walled, nonpigmented, wide $(5-20 \mu \mathrm{m})$, ribbon-like, pauciseptate (no or few septa), and have rightangle branching.(12)The fungi under the Mucorales are saprophytic and are ubiquitously present. Rhizopus arrhizus is the most common species affecting all over the world. Other isolates include Actinomucor, Cokeromyces, Syncephalastrum, Lichtheimia, Mucor, Rhizomucor, Cunninghame, Apophysomyces. Newer species that have been reported include Mucor irregularis, Thamnostylumlucknowense, Saksenaeaerythrospora. The species vary depending on the geographical area. Rhizopus, Mucor, Lichtheimia species cause $75 \%$ of cases.(13)Humans acquire the infection mainly by inhalation; other modes of transmission are by injection of the contaminated food and traumatic implantation (12).

\section{Risk factors}

Underlying diseases associated with Mucormycosis include diabetes mellitus, which is the most common risk factor in India, 
followed by haematological malignancy, organ transplant, prolonged neutropenia, corticosteroid therapy, diabetic ketoacidosis, trauma iron overload, deferoxamine therapy, and malnutrition. Other risk factors reported include chronic kidney disease, chronic obstructive lung disease, tuberculosis. Breakthrough Mucormycosis after voriconazole prophylaxis treatment have been documented in haematological malignancy patients (14).

\section{Clinical features}

Mucormycosis developed in patients with both active SAR-CoV infection and patients who had recovered from COVID19 infection (15). Infection was predominately seen in male patients. $80 \%$ had pre-existing diabetes mellites, $76.3 \%$ were treated with corticosteroids and $14.9 \%$ had diabetic ketoacidosis (15). Mucormycosis involving the nose and sinuses was the most common type.

Depending upon the site of involvement, clinically Mucormycosis is classified as rhinoorbital-cerebral (ROCM), pulmonary, gastrointestinal, cutaneous, disseminated Mucormycosis and othersinclude renal, osteomyelitis, endocarditis, peritonitis, ear, breast, uterus, urinary bladder, lymph nodes $(16,17)$. In India, ROCM is the most common type, followed by the pulmonary and cutaneous types (18). In developed countries, the pulmonary type is the most common. Multiple studies have shown that the type of Mucormycosis that a patient develops is associated with the underlying illness /predisposing factors.

Rhino-orbital-cerebral Mucormycosis is the most common type seen in patients with COVID-19 infection. It is often seen in patients with uncontrolled diabetes and diabetic ketoacidosis (13). Other risk factors include corticosteroid therapy, solid organ transplantation, intravenous drug use. Clinically the patient presents with fever, headache, facial swelling, facial swelling, pain, sinusitis, nasal discharge, epistaxis, nasal ulceration, ptosis, proptosis, eschars over the skin surface, or the palate, eye pain, diminished vision, orbital cellulitis. The infection can extend into the ethmoid, paranasal sinus, retro-orbitally into the cerebrum. This clinical type is observed with $46 \%$ of mortality (19).

Pulmonary Mucormycosis is the second most common clinical type. It is mainly seen in patients with haematological malignancy and organ transplant patients. It is also seen in patients with diabetes mellitus, hematopoietic stem cell transplant, post pulmonary tuberculosis patients (20). Pulmonary Mucormycosis is usually unilateral, and the upper lobe is the most affected (21). Clinically the patient presents with fever, breathing difficulty, chest pains, cough, pleuriticchest pain, and haemoptysis. This clinical type is associated with very high mortality of $76 \%$ (19).

Cutaneous Mucormycosis usually occurs following a breach in the epithelium following a trauma. This clinical type is often seen in immunocompetent patients. In a review by Skiada et al., 43-67\% of patients were immunocompetent (22). Depending on the depth of involvement, cutaneous Mucormycosis isclassified as follows (1) localized infection - the infection is limited to the kin and the subcutaneous tissues (2) deep extensions -there is the involvement of the muscle, tendons, bones (3) disseminated infection. Clinically they present with pain, swelling, ulcers, blisters, eschar formation.

Gastrointestinal Mucormycosis is usually seen in premature infants, patients undergoing peritoneal dialysis, patients with malnutrition. 
Symptoms include abdominal pain, nausea, vomiting and GI bleeding. A high index of suspicion is required to diagnose this clinical type.

Disseminate Mucormycosis has a very high mortality rate of $96 \%$ (19). Another rare type, is renal Mucormycosis which has been reported in immunocompetent patients in India and China (23).

\section{Pathophysiology}

\section{Virulence factors of Mucorales}

The pathogen's virulence depends on the ability of the organism to survive in the host by evading the immune system and causing harm to the host cells (24). Virulence factors of Mucorales include rapid multiplication, the ability to use the host iron, and its ability to attach to the endothelial surfaces.

High- affinity iron permease (FTR 1) helps in the uptake and transport of iron in the fungi. In immunodeficient mice, this gene is highly expressed (25). Conditional inactivation of the gene renders the fungi non-pathogenic in the mice and increases its survival rate. Antibodies against the FTR 1 induces protection against Mucormycosis infection in the mice (26).

Spore coat $(\mathrm{CotH})$ protein is present on the spore surface of the Mucorales. This protein binds to the GPR78 receptor on the endothelial cells.(27) It is an important virulence factor and plays a key role in the pathogenesis of Mucormycosis.

Alkaline Rhizopus protease enzyme (Arp) is another virulent factor that enhances the coagulation process in patients.(28)Other virulence factors include serine and aspartate proteases (SAPs), dihydrolipoyl dehydrogenase, ADP-ribosylation factor (Arf).

\section{Covid 19 and Mucormycosis}

During the pandemic, especially in the second wave, there is a dramatic increase in the Mucormycosis cases. This is well documented in multiple published case reports, case series, and review articles. The majority of the cases are reported from India. Diabetes mellites and use of systemic steroids are most common risk factors for Mucormycosis in the covid 19 patients.

COVID 19 is an infective inflammatory disease. It is clinically characterized by acute respiratory distress syndrome, pneumonia, and eventually multi-organ failure. The various pathogenic mechanism of covid 19 is being investigated and is still unfolding. Factors involved in the pathogenesis of COVID 19 which might be involved in the pathomechanism of Mucormycosis are (1) increased expression of GRP 78 receptors (2) alteration of iron metabolism - therole of ferritin and iron (3) hepcidin mimicking activity (4) endothelities (5) damage to beta islet cells of pancreas (6) use of corticosteroid.

Glucose-regulated protein 78 (GRP 78) is a host receptor present in the human endothelial cell, and it selectively and specifically interacts with the Mucorales. (29)GPR 78 receptor is also used by the SARS-CoV-2 virus for internalization into the endothelial cells.(30) Pathognomic feature of the Mucorales Is their ability to invade the blood vessels and cause thrombosis and tissue damage. Once the Mucorales spores enter the mucosa or skin, they bind to the laminin or the type IV collagen present in the basement membrane. Subsequently the CotH 3 on the spore, which is the fungal ligand, interacts with the GPR 78 in the host endothelium (27, 31). In COVID 19 infection, due to the interaction of the viral spike glycoprotein, there is an increase in the synthesis of the GRP 78. GPR 78 is a heat shock protein that is synthesized by the endothelial cells during 
stress (30). Multiple studies have shown, increased expression of GRP 78 increases the virulence of the Mucorales (27). GPR 78 expression increases in hyperglycaemia, diabetic ketoacidosis, Endothelitis, hyperferritinemia, and increased serum iron. The above factors are directly or indirectly related to the SARC-Cov-2 infection.

A study revealed increased serum iron is a risk factor for developing Mucormycosis.(32) FTR 1 is involved in iron uptake and transport in the Mucorales, as discussed under the virulence factor. In severe COVID 19, there is dysregulation in the iron homeostasis, and there is increased ferritin levels. $(33,34)$ In severe COVID 19 increase in the serum ferritin level occurs by two mechanisms, one ferritin is released into the circulation as a part of the inflammatory response, and secondarily because of hepcidin mimetic action of SARSCoV-2 $(35,36)$. High serum ferritin increases the intracellular iron, which generates reactive oxygen species, resulting in tissue damage. Furthermore, insevere COVID 19 due to an increase in macrophages activation and interleukin 16, stimulate the ferritin production, and the vicious cycle continues (36). Hepcidin is a crucial regulator of iron entry into the cell as it interacts with the ferroportin. It is primarily secreted by the liver. Due to the hepcidin mimetic activity of SAR-CoV-2, there is dysregulation of iron metabolism, which leads to hyperferritinemia.

Endothelitis occur in COVID 19 due to two possible mechanism: hyperferritinemia and free circulating dysfunctional heme (6). Heme is released into circulation by the interaction of the SAR-CoV-2 with haemoglobin via ACE 2, CD26, CD 417, and other receptors on the erythrocytes. After endocytosis of the viral particle into the erythrocytes, viral ORF8 protein interacts with the 1 beta chain of haemoglobin (37). This is subsequently released into the circulation as dysfunctional haemoglobin with reduced oxygen transport capability. This circulating free heme, iron, and ferritin causes free radical meditated endothelial inflammation leading to endothelities (34). In Endothelities there is upregulation of the GRP 78 receptors on the endothelial cell, this increases the virulence of the Mucorales by facilitating interaction between CotH3 and GPR78 (38).

Steroid therapy used in the treating COVID 19 infection, although recommended is a doubleedged sword because it predisposes patients to secondary Mucormycosis. There is an increased risk of Mucormycosis in diabetic patients who were started on corticosteroid to treat SAR-CoV-2 infection $(39,40)$. This can be attributed to the ability of the corticosteroid to decrease the immunity in the patients by impairing the migration, ingestion, and phagolysosome fusion in the macrophages. Steroid induced hyperglycaemia upregulates the expression of GPR 78, which in turn increases the pathogenicity and virulence of Mucorales (41).

Patients with COVID 19 infection are predisposed to develop diabetic keto acidosis. This is attributed to SARS CoV-1 mediated damage to pancreatic islet cells (42).

Heath care-related or nosocomial infection though rare can not be neglected. In a study conducted in India, $9 \%$ of cases of Mucormycosis were nosocomial.(2) Mucormycosis secondary to wooden tongue depressors, adhesives, bandages, insulin pump, the nitro-glycerine patch has been reported (43).

\section{Diagnosis}

Early diagnosis of Mucormycosis is essential, as it improves the outcome and decreases the need for surgical intervention hence disfigurement (44). Patients with suspected 
Mucormycosis should be referred to a higher centre with facilities for diagnosis and management of the complication. Routine laboratory diagnosis includes direct microscopy, culture and histopathology.

Direct microscopy is an inexpensive but valuable method for a rapid presumptive diagnosis of Mucormycosis. It is strongly recommended along with histopathology (45). Culture is essential as it allows identification of genus and species of Mucormycosis and antifungal susceptibility testing. A definitive diagnosis of Mucormycosis can be established by histopathological examination of the biopsies. Stains that help to highlight the Mucorales wall include periodic acid-Schiff stain, Grocott methenamine silvery stain.

Several molecular methods are emerging to detect the fungal DNA in serum and other body fluids, including PCR-based techniques.(46) But Currently, there is no standardized molecular-based method for the diagnosis of Mucormycosis. Many of the methods perform better in fresh or frozen samples than paraffin embedded samples.

\section{Management}

Treatment of Mucormycosis involves antifungal therapy, surgical debridement and management of underlying risk factors. Early diagnosis and initiation of treatment decreases the mortality associated with Mucormycosis. Mucormycosis can be effectively managed in facilities where surgical debridement and antifungal treatments are available.

The guidelines for managing Mucormycosis by the European confederation of medical oncology strongly recommend surgical debridement wherever possible along with antifungal therapy (45). Amphotericin B at the dose of 5-10 mg is the first drug of choice for Mucormycosis treatment with various organ involvement. Amphotericin B at a dose of 5 $\mathrm{mg} / \mathrm{kg}$ has been successfully used in the absence of CNS involvement. In CNS Involvement, liposomal amphotericin B at the dose of $5 \mathrm{mg} / \mathrm{kg}$ is recommended with moderate strength (45).

Posaconazole oral suspension and delayed released tablets are moderately supported.

Isavuconazole and Posaconazole are strongly recommended for salvage treatment. Antifungal treatment is continued until the infection is resolved.

Mucormycosis In COVID 19 can be prevented by (1) maintaining hygiene - regular change of mask, use of a disposable humidifier, using clean water for steaming and regular decontamination of the surfaces, (2) judicious use of steroid and immunosuppressants, (3) control of co-morbidities (47).

The surge in the Mucormycosis in COVID 19 infected patients can be explained by following factors involved in the pathogenesis of COVID 19 - increased expression of GRP 78 receptors, alteration of iron metabolism, hepcidin mimicking activity, endothelities, damage to beta islet cells of pancreas and use of corticosteroid. Further understanding of the pathogenesis helps in taking measures in the prevention and early management of the infection.

\section{References}

1. Chakrabarti A, Das A, Sharma A, Panda N, Das S, Gupta K L, Sakhuja V. Ten years' experience in zygomycosis at a tertiary care centre in India. J Infect. 2001 May;42(4):261-6.

2. Chakrabarti A, Chatterjee S S, Das A, Panda N, Shivaprakash M R, Kaur A, Varma S C, Singhi S, Bhansali A, Sakhuja V. Invasive zygomycosis in India: 
experience in a tertiary care hospital. Postgrad Med J. 2009 Nov;85(1009):57381.

3. Chakrabarti A, Singh R. Mucormycosis in India: unique features. Mycoses. 2014 Dec;57 Suppl 3:85-90.

4. Whiting D R, Guariguata L, Weil C, Shaw J. IDF diabetes atlas: global estimates of the prevalence of diabetes for 2011 and 2030. Diabetes Res Clin Pract. 2011 Dec;94(3):311-21.

5. Klomp, M, Ghosh, S, Mohammed, S, Khan, MN. From virus to inflammation, how influenza promotes lung damage. $J$ Leukoc Biol. 2021; 110: 115- 122.

6. Varg Z, Flammer A J, Steiger P, et al., Endothelial cell infection and endothelitis in COVID 19. Lancet 2020; 395: 1417-8.

7. Ehsani S. COVID-19 and iron dysregulation: distant sequence similarity between hepcidin and the novel coronavirus spike glycoprotein. Biol Direct. 2020 Oct 16;15(1):19.

8. Jose A, Singh S, Roychoudhury A, Kholakiya Y, Arya S, Roychoudhury S. Current Understanding in the Pathophysiology of SARS-CoV-2Associated Rhino-Orbito-Cerebral Mucormycosis: A Comprehensive Review. J Maxillofac Oral Surg. 2021 Jun 16:1-8.

9. La Fauci V, Costa G B, Facciolà A, Conti A, Riso R, Squeri R. Humidifiers for oxygen therapy: what risk for reusable and disposable devices? J Prev Med Hyg. 2017 Jun;58(2):E161-E165.

10. Rawson T M, Moore L S P, Zhu N, Ranganathan N, Skolimowska K, Gilchrist $\mathrm{M}$, et al., Bacterial and Fungal Coinfection in Individuals With Coronavirus: A Rapid Review To Support COVID-19 Antimicrobial Prescribing. Clin Infect Dis. 2020 Nov 1;71(9):2459-68.

11. Walther G, Wagner L, Kurzai O. Updates on the Taxonomy of Mucorales with an Emphasis on Clinically Important Taxa. J Fungi Basel Switz. 2019 Nov
14;5(4):E106.

12. Ribes J A, Vanover-Sams C L, Baker D J. Zygomycetes in human disease. Clin Microbiol Rev. 2000 Apr;13(2):236-301.

13. Jeong W, Keighley C, Wolfe R, Lee W L, Slavin MA, Kong DCM, et al., The epidemiology and clinical manifestations of mucormycosis: a systematic review and meta-analysis of case reports. Clin Microbiol Infect Off Publ Eur Soc Clin Microbiol Infect Dis. 2019 Jan;25(1):2634.

14. Trifilio S M, Bennett C L, Yarnold P R, McKoy J M, Parada J, Mehta J, et al., Break through zygomycosis after voriconazole administration among patients with hematologic malignancies who receive hematopoietic stem-cell transplants or intensive chemotherapy. Bone Marrow Transplant. 2007 Apr;39(7):425-9.

15. Singh A K, Singh R, Joshi S R, Misra A. Mucormycosis in COVID-19: A systematic review of cases reported worldwide and in India. Diabetes MetabSyndr. 2021 May 21;15(4):102146.

16. Kataria S P, Sharma J, Singh G, Kumar S, Malik S, Kumar V. Primary breast mucormycosis: FNAC diagnosis of a rare entity. Diagn Cytopathol. 2016 Sep;44(9):761-3.

17. Krishnappa D, Naganur S, Palanisamy D, Kasinadhuni G. Cardiac mucormycosis: a case report. Eur Heart J Case Rep. 2019 Sep 1;3(3):ytz130.

18. Patel A, Kaur H, Xess I, Michael J S, Savio J, Rudramurthy S, Singh R, Shastri $\mathrm{P}$, Umabala $\mathrm{P}$, Sardana R, Kindo A, Capoor M R, Mohan S, Muthu V, Agarwal R, Chakrabarti A. A multicentre observational study on the epidemiology, risk factors, management and outcomes of mucormycosis in India. Clin Microbiol Infect. $2020 \mathrm{Jul} ; 26(7): 944 . e 9-944 . e 15$.

19. Roden M M, Zaoutis T E, Buchanan W L, Knudsen T A, Sarkisova T A, Schaufele R 
L, et al., Epidemiology and Outcome of Zygomycosis: A Review of 929 Reported Cases. Clin Infect Dis. 2005 Sep 1;41(5):634-53.

20. Prakash H, Ghosh A K, Rudramurthy S M, Singh P, Xess I, Savio J, et al., A prospective multicenter study on mucormycosis in India: Epidemiology, diagnosis, and treatment. Med Mycol. 2019 Jun 1;57(4):395-402.

21. Lee F Y, Mossad S B, Adal K A. Pulmonary mucormycosis: the last 30 years. Arch Intern Med. 1999 Jun 28;159(12):1301-9.

22. Feng J, Sun $X$. Characteristics of pulmonary mucormycosis and predictive risk factors for the outcome. Infection. 2018 Aug;46(4):503-12.

23. Bhadauria D, Etta P, Chelappan A, Gurjar M, Kaul A, Sharma RK, et al., Isolated bilateral renal mucormycosis in apparently immunocompetent patients - a case series from India and review of the literature. Clin Kidney J. 2018 Dec;11(6):769-76.

24. Brunke S, Mogavero S, Kasper L, Hube B. Virulence factors in fungal pathogens of man. CurrOpinMicrobiol. 2016 Aug;32:89-95.

25. Navarro-Mendoza MI, Pérez-Arques C, Murcia L, Martínez-García P, Lax C, Sanchis M, et al., Components of a new gene family of ferroxidases involved in virulence are functionally specialized in fungal dimorphism. Sci Rep. 2018 May 16;8:7660.

26. Ibrahim A S, Gebremariam T, Lin L, Luo G, Husseiny M I, Skory C D, et al., The high affinity iron permease is a key virulence factor required for Rhizopus oryzae pathogenesis. Mol Microbiol. 2010 Aug;77(3):587-604.

27. Gebremariam T, Liu M, Luo G, Bruno V, Phan Q T, Waring A J, et al., CotH3 mediates fungal invasion of host cells during mucormycosis. J Clin Invest. 2014 Jan 2;124(1):237-50.
28. Spreer A, Rüchel R, Reichard U. Characterization of an extracellular subtilisin protease of Rhizopus microsporus and evidence for its expression during invasive rhinoorbital mycosis. Med Mycol. 2006 Dec;44(8):723-31.

29. Liu M, Spellberg B, Phan Q T, Fu Y, Fu Y, Lee A S, Edwards J E Jr, Filler S G, Ibrahim A S. The endothelial cell receptor GRP78 is required for mucormycosis pathogenesis in diabetic mice. J Clin Invest. $2010 \mathrm{Jun} ; 120(6): 1914-24$.

30. Ibrahim I M, Abdelmalek D H, Elshahat M E, Elfiky A A. COVID-19 spike-host cell receptor GRP78 binding site prediction. J Infect. 2020 May;80(5):554562.

31. Bouchara J P, Oumeziane N A, Lissitzky J C, Larcher G, Tronchin G, Chabasse D. Attachment of spores of the human pathogenic fungus Rhizopus oryzae to extracellular matrix components. Eur J Cell Biol. 1996 May;70(1):76-83.

32. Ibrahim A, Spellberg B, Edwards J. Iron Acquisition: A Novel Prospective on Mucormycosis Pathogenesis and Treatment. CurrOpin Infect Dis. 2008 Dec;21(6):620-5.

33. Perricone C, Bartoloni E, Bursi R, Cafaro G, Guidelli GM, Shoenfeld Y, et al., COVID-19 as part of the hyperferritinemic syndromes: the role of iron depletion therapy. Immunol Res. 2020;68(4):21324.

34. John T M, Jacob C N, Kontoyiannis D P. When Uncontrolled Diabetes Mellitus and Severe COVID-19 Converge: The Perfect Storm for Mucormycosis. J Fungi Basel Switz. 2021 Apr 15;7(4):298.

35. Cavezzi A, Troiani E, Corrao S. COVID19: hemoglobin, iron, and hypoxia beyond inflammation. A narrative review. Clin Pract. 2020 May 28;10(2):1271.

36. Colafrancesco S, Alessandri C, Conti F, Priori R. COVID-19 gone bad: A new 
character in the spectrum of the hyperferritinemic syndrome? Autoimmun Rev. 2020 Jul;19(7):102573.

37. Wenzhong L, Hualan L. COVID-19: captures iron and generates reactive oxygen species to damage the human immune system. Autoimmunity. 2021 Jun;54(4):213-224.

38. Baldin C, Ibrahim A S. Molecular mechanisms of mucormycosis-The bitter and the sweet. PLoSPathog. 2017 Aug 3;13(8):e1006408.

39. Moorthy A, Gaikwad R, Krishna S, Hegde R, Tripathi K K, Kale PG, et al., SARSCoV-2, Uncontrolled Diabetes and Corticosteroids-An Unholy Trinity in Invasive Fungal Infections of the Maxillofacial Region? A Retrospective, Multi-centric Analysis. J Maxillofac Oral Surg. 2021 Mar 6;1-8.

40. Ahmadikia K, Hashemi S J, Khodavaisy S, Getso M I, Alijani N, Badali H, Mirhendi H, Salehi M, Tabari A, Mohammadi Ardehali M, Kord M, Roilides E, Rezaie S. The double-edged sword of systemic corticosteroid therapy in viral pneumonia: A case report and comparative review of influenza-associated mucormycosis versus COVID-19 associated mucormycosis. Mycoses. 2021 Aug;64(8):798-808

41. Bonaventura A, Montecucco F. Steroidinduced hyperglycemia: An under diagnosed problem or clinical inertia? A narrative review. Diabetes Res Clin Pract. 2018 May;139:203-20.
42. Yang J K, Lin S S, Ji X J, Guo L M. Binding of SARS coronavirus to its receptor damages islets and causes acute diabetes. Acta Diabetol. 2010 Sep;47(3):193-9.

43. Rammaert B, Lanternier F, Zahar J-R, Dannaoui E, Bougnoux M-E, Lecuit M, et al., Healthcare-associated mucormycosis. Clin Infect Dis Off Publ Infect Dis Soc Am. 2012 Feb;54 Suppl1:S44-54.

44. Chamilos G, Lewis R E, Kontoyiannis D P. Delaying amphotericin B-based frontline therapy significantly increases mortality among patients with hematologic malignancy who have zygomycosis. Clin Infect Dis Off Publ Infect Dis Soc Am. 2008 Aug 15;47(4):503-9.

45. Cornely O A, Alastruey-Izquierdo A, Arenz D, Chen S C A, Dannaoui E, Hochhegger $\mathrm{B}$, et al., Global guideline for the diagnosis and management of mucormycosis: an initiative of the European Confederation of Medical Mycology in cooperation with the Mycoses Study Group Education and Research Consortium. Lancet Infect Dis. 2019 Dec;19(12):e405-21.

46. Dadwal S S, Kontoyiannis D P. Recent advances in the molecular diagnosis of mucormycosis. Expert Rev Mol Diagn. 2018 Oct;18(10):845-54.

47. Ish P, Ish S. Prevention of mucormycosis in COVID-19 - the need of the hour. Indian J Ophthalmol. 2021 Jul 1;69:1969.

\section{How to cite this article:}

Vijayalakshmi Nayak and Shyamala, R. 2021. Pernicious Combination of Covid-19 and Mucormycosis. Int.J.Curr.Microbiol.App.Sci. 10(08): 620-628. doi: https://doi.org/10.20546/ijcmas.2021.1008.072 